following initiation of internal quality improvement (QI) initiative at St Louis University Hospital (SLUH).

Materials and Methods All patients who received IV-tPA or MT at SLUH from December 2016 to May 2018 and July 2018 to December 2019 were included in this study and dichotomized into 'Pre-intervention' and 'Post-intervention' groups. Chart review data including patient demographics, arrival method, and risk factors were collected retrospectively. In addition, relevant times were collected which included time of ED arrival, time of NIHSS, time of CT acquisition, time of tPA bolus, time of groin puncture, and time of recanalization. NIHSS at discharge and complications of therapy were also collected.

Results For those receiving tPA, mean time to NIHSS was similar in the pre- and post-intervention groups, 4.28 minutes and 3.88 minutes, respectively $(t=0.25, p=0.80)$; mean time to CT acquisition was also similar, 11.28 minutes and 12.53 minutes $(t=-0.53, p=0.60)$. However, mean time for DTN decreased from 42.52 minutes to 33.87 minutes following the quality improvement initiative $(t=2.29$, $\mathrm{p}=0.02)$. tPA post-intervention patients were less likely to have asymptomatic ICH $\left(\chi^{2}=6.22, p=0.01\right)$ and less likely to have other complications $\left(\chi^{2}=4.66, p=0.03\right)$. For those receiving MT, mean time to NIHSS similar in both groups, 3.81 minutes compared to 5.55 minutes in the post-intervention group $(\mathrm{t}=-0.66, \mathrm{p}=0.51)$; mean time to CT acquisition was 10.53 minutes compared to 12.23 minutes $(t=-0.52$, $\mathrm{p}=0.60)$. DGPT decreased from 101.81 minutes to 75.91 minutes $(t=3.48, p=0.001)$ and mean time to recanalization decreased from 176.89 minutes to 109.74 minutes $(t=6.68$, $\mathrm{p}<0.001)$. In the MT group, no significant differences were found in complication rates between the pre- and post-intervention groups.

Conclusion Our internal QI Initiative to improve workflow latencies in the Code Stroke Protocol resulted in statistically significant reductions in DTN and DPGTs.

Disclosures V. Chin: None. K. Yeboah: 5; C; Saint Louis University. A. Balushi: None. A. Guthrie: 5; C; Saint Louis University. K. Christopher: 5; C; Saint Louis University. R. Edgell: 5; C; Saint Louis University.

\section{E-069 PREVENTABILITY OF STROKE DUE TO LARGE VESSEL OCCLUSION TREATED WITH MECHANICAL THROMBECTOMY}

${ }^{1} \mathrm{~S}$ Suzuki ${ }^{*},{ }^{1} \mathrm{~B}$ Nguyen, ${ }^{1} \mathrm{I}$ Yuki, ${ }^{1} \mathrm{~J} \mathrm{Xu},{ }^{2} \mathrm{M}$ Fisher. ${ }^{1}$ Neurological Surgery, University of California Irvine, Orange, $C A ;{ }^{2}$ Neurology, University of California Irvine, Orange, $C A$

10.1136/neurintsurg-2020-SNIS.105

Background and Objective Mechanical Thrombectomy (MT) is standard treatment for acute ischemic stroke due to large vessel occlusion (LVO). However, extent of preventability of these strokes is generally not addressed. This study analyzes stroke preventability for patients treated with MT for LVO.

Subjects and Methods We conducted retrospective analyses of stroke patients treated with MT for LVO at UC Irvine Medical Center from June 2008 to March 2019. We obtained data including age, NIH Stroke Scale (NIHSS) at onset, and (beginning in 2015) 90-day outcome measured by modified Rankin Scale (mRS). We used the Stroke Preventability Score (SPS, 010 points) from our prior work (JAMA Neurology 2016, 73:186), based on how well patients had been treated given their hypertension, hyperlipidemia, atrial fibrillation, and prior stroke history. We examined associations of SPS with age, NIHSS at onset, and mRS.

Results For 301 patients treated with MT for LVO, mean \pm standard error of age was $60 \pm 0.1$ years (range 18-97) and NIHSS was $17 \pm 0.4$ (range $0-37, n=282$ ); one-third $(n=96)$ were male. SPS was calculated for 273 patients, with mean \pm SE 2.1 \pm 0.1 (range 0-8). Over two-thirds ( $n=184,67 \%$ ) demonstrated some degree of preventability (SPS 1 or higher), while 23\% ( $n=64)$ showed high stroke preventability (SPS 4 or higher). Among those with high SPS, atrial fibrillation with no treatment was seen in 54 (85.7\%) patients. Among 119 patients with mRS data, stroke preventability was associated with age $(66 \pm 2.5,69 \pm 2.4$ and $79 \pm 1.7$ years for no, low and high SPS, respectively; $\mathrm{p}<0.002)$. Stroke preventability was also associated with NIHSS at onset (15 $\pm 1.3,16 \pm 0.9$, and $20 \pm 1.4$ for no, low and high preventability, respectively; $\mathrm{p}<0.04)$. SPS was significantly correlated with age $(\mathrm{r}=0.33, \mathrm{p}<0.0002)$, NIHSS $(\mathrm{r}=0.29, \mathrm{p}=0.002)$, and mRS $(r=0.22, p=0.02)$. The partial correlation coefficient for SPS remained significant after adjusting for age and NIHSS.

Conclusion Nearly one-quarter of patients, with LVO treated with MT, presented with strokes that were highly preventable. High stroke preventability tended to be seen in older patients, patients with more severe deficits, and (to a lesser extent) patients with poor outcome. Severe LVO strokes with the worst outcome (post-mechanical thrombectomy) tended to be the most preventable strokes. While most acute stroke treatment efforts focus on streamlining workflow, these findings demonstrate the enduring importance of stroke prevention in this patient population. These findings raise the question whether resources for treatment of acute stroke are being directed toward patients whose strokes are the most preventable.

Disclosures S. Suzuki: None. B. Nguyen: None. I. Yuki: None. J. Xu: None. M. Fisher: None.

\section{E-070 ENDOVASCULAR TREATMENT OF THE VERTEBRAL ARTERY ORIGIN STENOSIS BY USING THE CLOSED-CELL, SELF-EXPANDABLE CAROTID WALLSTENT}

1J KO*, ${ }^{2} \mathrm{~T}$ Lee. ${ }^{1}$ Neurosurgery, Pusan National University Hospital, Busan, Korea, Republic of, ${ }^{2}$ Diagnostic Radiology, Pusan National University Hospital, Busan, Korea, Republic of

\subsection{6/neurintsurg-2020-SNIS. 106}

Background Endovascular treatment has been considered a good alternative to surgery for symptomatic vertebral artery origin stenosis (VAOS) due to the high risk of morbidity associated with surgery. The purpose of this study was to evaluate the feasibility and efficacy of insertion of the closed-cell, selfexpandable Carotid Wallstent for treatment of VAOS.

Methods The records of 72 patients with VAOS refractory to adequate medication who were treated by endovascular treatment with the Carotid Wallstent from December 2004 to November 2010 were retrospectively evaluated.

Results Of the 72 patients, 43 presented with transient ischemic attacks. Forty-seven patients (65.3\%) manifested other brachiocephalic stenoses; of these, 40 patients had occlusion, hypoplasia, or stenosis of the contralateral vertebral artery. Overall technical success (defined as $20 \%$ or less residual stenosis) was $100 \%$. Procedure-related complications $(n=8$, 